Article

\title{
In Vitro Activity of 22 Antibiotics against Achromobacter Isolates from People with Cystic Fibrosis. Are There New Therapeutic Options?
}

\author{
Clémence Beauruelle ${ }^{1,2, *} \mathbb{C}$, Claudie Lamoureux ${ }^{1,2}{ }^{2}$ Arsid Mashi ${ }^{2}$, Sophie Ramel ${ }^{3}$, Jean Le Bihan ${ }^{3}$, \\ Thomas Ropars ${ }^{3}$, Anne Dirou ${ }^{3}$, Anandadev Banerjee ${ }^{3}$, Didier Tandé ${ }^{2}$, Hervé Le Bars ${ }^{2}$ \\ and Geneviève Héry-Arnaud ${ }^{1,2}$ (D) \\ 1 University Brest, INSERM, EFS, UMR 1078, GGB, 29200 Brest, France; claudie.lamoureux@chu-brest.fr (C.L.); \\ Genevieve.Hery-Arnaud@univ-brest.fr (G.H.-A.) \\ 2 Department of Bacteriology, Virology, Hospital Hygiene, and Parasitology-Mycology, \\ Brest University Hospital, 29200 Brest, France; arsid.mashi@gmail.com (A.M.); \\ didier.tande@chu-brest.fr (D.T.); herve.lebars@chu-brest.fr (H.L.B.) \\ 3 Centre de Ressources et de Compétences de la Mucoviscidose, Fondation Ildys, Presqu'île de Perharidy, \\ 29680 Roscoff, France; sophie.ramel@ildys.org (S.R.); jean.lebihan@ildys.org (J.L.B.); \\ thomas.ropars@ildys.org (T.R.); anne.dirou@ildys.org (A.D.); anandadev.banerjee@ildys.org (A.B.) \\ * Correspondence: clemence.beauruelle@chu-brest.fr; Tel.:+332-98-14-51-05
}

check for updates

Citation: Beauruelle, C.; Lamoureux, C.; Mashi, A.; Ramel, S.; Le Bihan, J.; Ropars, T.; Dirou, A.; Banerjee, A.; Tandé, D.; Le Bars, H.; et al. In Vitro Activity of 22 Antibiotics against Achromobacter Isolates from People with Cystic Fibrosis. Are There New Therapeutic Options? Microorganisms 2021, 9, 2473. https://doi.org/ 10.3390 /microorganisms 9122473

Academic Editor: Lucie Amoureux

Received: 30 September 2021

Accepted: 26 November 2021

Published: 30 November 2021

Publisher's Note: MDPI stays neutral with regard to jurisdictional claims in published maps and institutional affiliations.

Copyright: (c) 2021 by the authors. Licensee MDPI, Basel, Switzerland. This article is an open access article distributed under the terms and conditions of the Creative Commons Attribution (CC BY) license (https:// creativecommons.org/licenses/by/ $4.0 /)$.
Abstract: Bacteria belonging to the genus Achromobacter are increasingly isolated from respiratory samples of people with cystic fibrosis (PWCF). The management of this multidrug-resistant genus is challenging and characterised by a lack of international recommendations, therapeutic guidelines and data concerning antibiotic susceptibility, especially concerning the newer antibiotics. The objective of this study was to describe the antibiotic susceptibility of Achromobacter isolates from PWCF, including susceptibility to new antibiotics. The minimum inhibitory concentrations (MICs) of 22 antibiotics were determined for a panel of 23 Achromobacter isolates from 19 respiratory samples of PWCF. Two microdilution MIC plates were used: EUMDROXF ${ }^{\circledR}$ plate (Sensititre) and Micronaut-S Pseudomonas MIC $^{\circledR}$ plate (Merlin) and completed by a third method if necessary (E-test ${ }^{\circledR}$ or UMIC ${ }^{\circledR}$ ). Among usual antimicrobial agents, the most active was imipenem (70\% susceptibility). Trimethoprimsulfamethoxazole, piperacillin and tigecycline $(65 \%, 56 \%$ and $52 \%$ susceptibility, respectively) were still useful for the treatment of Achromobacter infections. Among new therapeutic options, $\beta$-lactams combined with a $\beta$-lactamase-inhibitor did not bring benefits compared to $\beta$-lactam alone. On the other hand, cefiderocol appeared as a promising therapeutic alternative for managing Achromobacter infections in PWCF. This study provides the first results on the susceptibility of clinical Achromobacter isolates concerning new antibiotics. More microbiological and clinical data are required to establish the optimal treatment of Achromobacter infections.

Keywords: Achromobacter; non-fermenting Gram-negative bacilli; cystic fibrosis; antibiotic susceptibility; resistance; multidrug resistance

\section{Introduction}

Bacteria belonging to the genus Achromobacter are increasingly isolated from respiratory samples of people with cystic fibrosis (PWCF). In France, their prevalence raised from $3.1 \%$ in 1999 to $6.9 \%$ in 2019 [1]. This opportunistic pathogen has been associated with acute pulmonary exacerbations, chronic infections and higher concentrations of TNF- $\alpha$ in sputum samples. Achromobacter infection occurs most commonly in CF patients with advanced lung disease and is associated with a poor clinical course [2-7]. Achromobacter species are well-armed for colonisation and persistence in the CF lung, as they harbour a large panel of adaptive strategies (biofilm formation, antibiotic resistance, hypermutation, secretion and quorum sensing systems) [8]. However, the current evidence is insufficient 
to attribute a major role in disease progression and large prospective studies assessing the pathogenicity of this emerging pathogen are warranted. Today, there is no international standard concerning the management of Achromobacter lung infection (when to treat and how to treat), including in PWCF. Moreover, the introduction of an adapted antibiotic therapy is often difficult because of the numerous natural and acquired resistances that characterise the Achromobacter genus [9]. Resistance, either innate or acquired, is conferred by two major mechanisms: extrusion of the antibiotics through efflux pumps and enzymatic degradation [8,9]. At least two efflux pumps confer intrinsic resistances: the $A x y A B M$ efflux pump, which is mainly involved in the extrusion of cephalosporins, and the AxyXY-OprZ efflux pump, which is mainly involved in the extrusion of aminoglycosides. Achromobacter produces a constitutive $\beta$-lactamase (OXA-114) with activity against penicillin $\mathrm{G}$, ticarcillin, piperacillin and cephalotin even though ticarcillin and pipercacillin susceptibility is common among Achromobacter isolates. The lack of therapeutic guidelines was accompanied, until recently, by a lack of technical guidelines for performing antibiotic susceptibility tests, including specific breakpoints. Consequently, most of the published data are based on the CLSI (Clinical and Laboratory Standards Institute) breakpoints for the "other non-Enterobacterales" category, which is not adapted to characterise Achromobacter susceptibility. In 2020, EUCAST (European Committee on Antimicrobial Susceptibility Testing) proposed a genus-specific susceptibility testing method and consensually accepted susceptibility breakpoints for three antibiotics (i.e., piperacillin-tazobactam, meropenem and trimethoprim-sulfamethoxazole [SXT]) [10]. Another recent change concerns the availability of new antibiotics. Indeed, new antibiotics with anti-Gram negative activity (as cefiderocol, eravacycline or new $\beta$-lactam- $\beta$-lactamase inhibitor associations) are recently available and could be of interest in the treatment of Achromobacter infections.

Today, few data exist to describe the susceptibility of Achromobacter isolates to antibiotics, especially to the recently marketed molecules. Given this observation, the objective of our study was to describe the antibiotic susceptibility of Achromobacter in PWCF, including concerning new molecules. Therefore, we determined the minimum inhibitory concentration (MICs) of 22 antibiotics for a panel of 23 Achromobacter clinical isolates from respiratory samples of PWCF.

\section{Materials and Methods}

\subsection{Patients and Isolates Characteristics}

Twenty-three isolates of Achromobacter cultured from sputum of 19 PWCF followed at the Western Brittany CF centre (Roscoff, France) during the year 2020 have been included in this study. Sputum samples were collected during follow-up consultations as part of routine patient monitoring. Demographic data (sex and age) and state of Achromobacter colonisation were recorded. Patients were considered chronically colonised according to the criteria defined by Pereira [11] (at least three positive cultures in one year obtained with a minimum interval of one month between them, for at least two years).

Microbial analysis was performed at the clinical bacteriology unit of Brest University Hospital following French recommendations [12]. Isolates identified as Achromobacter by matrix-assisted laser desorption/ionisation time of flight mass spectrometry (MALDI-TOF MS biotyper MBT with the Bruker's library version 9 (Bruker, Bremen, Germany) were stored at $-80{ }^{\circ} \mathrm{C}$ (Microbank, Pro-Lab Diagnostics, Richmond Hill, ON, Canada), and grown secondarily on Columbia agar with 5\% horse blood (bioMérieux, Marcy l'Etoile, France). Freshly cultured isolates were spotted in duplicates onto MALDI-TOF MS target plates. A score of $\geq 1.7$ was considered as an accurate genus-level identification. As MALDITOF MS is not reliable at distinguishing Achromobacter at the species level, we did not consider the species identified by MALDI-TOF [13].

\subsection{Antibiotic Susceptibility Testing}

Susceptibility testing of the isolates was performed as recommended by EUCAST 2021 [14]. Briefly, an inoculum of $0.5 \mathrm{McFarland}$ was prepared in physiological water from a 
fresh culture and was used for the preparation of all the tests. The bacterial suspension was transferred in a cation-adjusted Mueller-Hinton (MH) broth (Merlin Diagnostika, GmbH, Bornheim, Germany). MIC plates based on microdilution were inoculated according to the manufacturer's recommendations: EUMDROXF ${ }^{\circledR}$ plate (Sensititre, Thermo Fisher Scientific, Cleveland, OH, USA) and Micronaut-S Pseudomonas $\mathrm{MIC}^{\circledR}$ plate (Merlin Diagnostika, $\mathrm{GmbH}$, Bornheim, Germany). The MICs of 22 antibiotics active on non-fermenting Gramnegative bacilli, listed in Table 1, were thus determined using the broth microdilution method. Given the dilution range for SXT contained in the plates, this association was also tested by the agar diffusion method. An MH agar plate (BioMerieux, Marcy-1'Etoile, France) was inoculated, and a disk of SXT (Bio-Rad, Marne-la-coquette, France) was tested, according to the EUCAST standardised disk diffusion method [15].

The MICs were determined as the lowest antibiotic concentration that completely inhibited visible bacterial growth after $20 \pm 4 \mathrm{~h}$ incubation at $35 \pm 2{ }^{\circ} \mathrm{C}$ in an aerobic atmosphere. $\mathrm{MIC}_{50}$ and $\mathrm{MIC}_{90}$ were calculated for the 22 antimicrobial agents [16]. The $\mathrm{MIC}_{50}$ and the $\mathrm{MIC}_{90}$ represent the MIC value at which $\geq 50 \%$ and $\geq 90 \%$ of the isolates are inhibited, respectively. Inhibition zone diameter was determined as the point where no obvious growth was detected or at the outer zone edge in case of a fine haze in the inhibition zone, following EUCAST recommendations [14]. All results were read in duplicate, independently, by two different people. In the case of a discrepancy between the two operators, a third person read the results. Pseudomonas aeruginosa ATCC 27853 was used as a control for the two microdilution MIC plates.

Results were interpreted according to EUCAST 2021 breakpoints for A. xylosoxidans for piperacillin-tazobactam, meropenem and SXT, for Pseudomonas for colistin and according to non-species-related pharmacokinetic/pharmacodynamic (PK/PD) breakpoints for the other antibiotics except for eravacycline for which there are no breakpoints. For eravacycine, the breakpoint for Enterobacterales was used (listed in Table S1).

Results were also interpreted according to CLSI 2021 breakpoints for other nonEnterobacterales and for P. aeruginosa in the absence of breakpoints (for ceftazidime-avibactam, ceftolozane-tazobactam, imipenem-relebactam, cefiderocol and colistin) (Table S1) [17].

\subsection{Comparison of Different Susceptibility Testing Methods}

We measured the agreement between the two methods calculating the Cohen's kappa ( $\kappa)$ considering susceptibility criteria according to EUCAST breakpoints [18]. The values of $\mathrm{K}$ were interpreted according to the method of Landis and Koch, as follows: 1.00 to 0.81 , excellent; 0.80 to 0.61 , good (substantial); 0.60 to 0.41 , moderate; 0.40 to 0.21 , fair; 0.20 to 0.00, negligible agreement [19].

A mismatch between plates was considered acceptable if it involved only one dilution gap and unacceptable if it involved two or more dilutions gaps. In the case of unacceptable mismatch, a third method was used (MIC microdilution by UMIC ${ }^{\circledR}$ (Biocentric, Bandol, France) for colistin or E-test ${ }^{\circledR}$ (bioMerieux, Marcy-l'Etoile, France) for other antimicrobial agents).

\section{Results}

\subsection{Patients and Isolates Characteristics}

A total of 23 isolates of Achromobacter cultured from sputum of 19 PWCF have been included. Among the 19 PWCF, 11 were chronically colonised, 4 were sporadically colonised and for the other four patients, the number of samples was insufficient to qualify patient colonisation. The sex ratio $\mathrm{M} / \mathrm{W}$ was 1.7 , and the median age was 27 years (range 9-47).

For four of the $19 \mathrm{PWCF}$, two strains were isolated simultaneously and were included in the study. 


\subsection{Antibiotic Susceptibility Testing}

The susceptibility testing results are summarised in Table 1 , as well as $\mathrm{MIC}_{50}$ and $\mathrm{MIC}_{90}$ for each antimicrobial agent. The full MIC distributions for these isolates are available in Table S2.

Table 1. In vitro activities of antimicrobial agents against Achromobacter isolates.

\begin{tabular}{|c|c|c|c|c|c|c|}
\hline \multirow[b]{3}{*}{ Antimicrobial agent } & \multirow{2}{*}{\multicolumn{2}{|c|}{$\begin{array}{c}\text { MIC (mg/L) } \\
50 \% / 90 \%\end{array}$}} & \multicolumn{4}{|c|}{$\begin{array}{c}\text { Percentage of Susceptibility According to the Following } \\
\text { Breakpoints }\end{array}$} \\
\hline & & & \multicolumn{2}{|c|}{ EUCAST $^{\mathrm{c}}$} & \multicolumn{2}{|c|}{ CLSI $^{d}$} \\
\hline & Merlin $^{a}$ & Sensititre $^{b}$ & Merlin $^{a}$ & Sensititre $^{b}$ & Merlin $^{a}$ & Sensititre ${ }^{b}$ \\
\hline Piperacillin & $\leq 4 />32$ & - & 57 & - & 61 & - \\
\hline Piperacillin—tazobactam & $4 />128$ & $\leq 4 />32$ & 57 & 57 & 65 & 65 \\
\hline Aztreonam & $>16 />16$ & $>32 />32$ & 0 & 0 & 0 & 0 \\
\hline Cefepime & $>8 />8$ & $>16 />16$ & 4 & 9 & 13 & 22 \\
\hline Ceftazidime & $>32 />32$ & - & 17 & - & 30 & - \\
\hline Ceftazidime-avibactam & $>8 />8$ & $16 />16$ & 26 & 39 & 26 & 39 \\
\hline Ceftolozane - tazobactam & $>8 />8$ & $>8 />8$ & 0 & 9 & 0 & 9 \\
\hline Cefiderocol & - & $0.25 / 1$ & - & 91 & - & 91 \\
\hline Imipenem & $\leq 1 />8$ & $\leq 1 / 2$ & 70 & 91 & 70 & 91 \\
\hline Imipenem-relebactam & - & $1 / 2$ & - & 91 & - & 91 \\
\hline Meropenem & $2 />16$ & $2 />16$ & 48 & 43 & 70 & 57 \\
\hline $\begin{array}{l}\text { Meropenem- } \\
\text { vaborbactam }\end{array}$ & - & $1 / 16$ & - & 87 & - & NA \\
\hline Ciprofloxacin & $4 / 8$ & - & 0 & - & 4 & - \\
\hline Levofloxacin & $4 / 8$ & - & 4 & - & 48 & - \\
\hline Colistin & $8 />8$ & $16 />16$ & 39 & 30 & 39 & 30 \\
\hline Fosfomycin & $>128 />128$ & $>64 />64$ & 0 & 0 & NA & NA \\
\hline Gentamicin & $>32 />32$ & - & 0 & - & 9 & - \\
\hline Amikacin & $>32 />32$ & $>32 />32$ & 0 & 0 & 22 & 13 \\
\hline Tobramycin & $32 />32$ & $>4 />4$ & 0 & 0 & 9 & 13 \\
\hline $\mathrm{SXT}^{\mathrm{e}}$ & $\leq 1 />8$ & - & 65 & - & 65 & - \\
\hline Tigecycline & - & $\leq 0.5 / 1$ & - & 52 & - & NA \\
\hline Eravacycline & - & $0.5 />0.5$ & - & 65 & - & NA \\
\hline
\end{tabular}

The MICs were determined using two MIC plates in microdilution: ${ }^{a}$ EUMDROXF ${ }^{\circledR}$ plate (Sensititre) and, ${ }^{\mathrm{b}}$ Micronaut-S Pseudomonas $\mathrm{MIC}^{\circledR}$ plate (Merlin). ${ }^{\mathrm{C}} \mathrm{MIC}$ were interpreted according to EUCAST breakpoints defined for A. xylosoxidans for piperacillin-tazobactam, meropenem and trimethoprim-sulfamethoxazole (SXT), for Pseudomonas for colistin, for Enterobacterales for eravacycin and according to pharmacokinetic/pharmacodynamic (non-species-related) EUCAST breakpoints for the other antibiotics. ${ }^{d}$ Results were interpreted according to CLSI breakpoint defined for other non Enterobacterales and for P. aeruginosa in the absence of breakpoints (for ceftazidime-

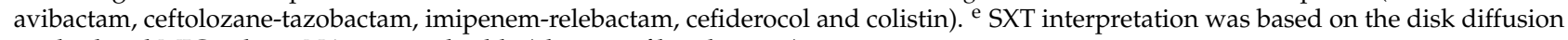
method and MIC values. NA: not applicable (absence of breakpoints).

\subsubsection{Percentage of Susceptibility According to EUCAST Breakpoints}

The 23 isolates included in the study displayed the innate resistance to aztreonam, aminoglycosides and fosfomycin expressed by the majority of Achromobacter species (Table 1).

Among the 22 antibiotics tested, the most active were cefiderocol (91\% susceptibility with both plates) and imipenem ( $70 \%$ and $91 \%$, respectively, with Merlin and Sensititre plates). Meropenem was less active than imipenem (respectively $48 \mathrm{vs.} 70 \%$ [Merlin] and 43 vs. $91 \%$ [Sensititre]). The association meropenem-vaborbactam was active for $87 \%$ of isolates. Piperacillin alone or associated with tazobactam was active in more than half of the isolates $(56 \%)$, as well as SXT $(65 \%)$ and tigecycline $(52 \%)$. Colistin remained susceptible for about one third of isolates (30\% [Sensititre] to 39\% [Merlin]). Resistance to ceftazidime was observed for the majority of isolates (17\% susceptibility). The addition of avibactam raised the susceptibility to $26 \%$. Cefepime, ceftolozane-tazobactam and fluoroquinolones were inactive for the majority of isolates ( $\leq 10 \%$ susceptibility) (Table 1$)$.

The addition of a $\beta$-lactamase inhibitor to a $\beta$-lactam appeared to provide a significant improvement for ceftazidime and meropenem with a raised susceptibility with avibactam 
and vaborbactam, respectively (17 to $26 \%$ for ceftazidime \pm avibactam and 43 to $87 \%$ for meropenem \pm vaborbactam). Nonetheless, $\mathrm{MIC}_{50}$ and $\mathrm{MIC}_{90}$ observed for ceftazidime or meropenem alone were similar or slightly different and generally concerned one MIC dilution to those obtained for ceftazidime-avibactam or meropenem-vaborbactam (Table 1 and Table S2). Concerning other $\beta$-lactams (piperacillin and imipenem), $\mathrm{MIC}_{50 / 90}$, percentage of susceptibility and MIC for each isolate were similar for $\beta$-lactam alone and $\beta$-lactam$\beta$-lactamase inhibitor association (piperacillin-tazobactam or imipenem-relebactam). We can also notice a breakpoint difference between $\beta$-lactam alone and $\beta$-lactam inhibitors for these antibiotics (Table S1), which can explain susceptibility differences.

Among new antimicrobial agents, eravacycline presented relatively low MICs $\left(\mathrm{MIC}_{50 / 90}=0.5 />0.5 \mathrm{mg} / \mathrm{L}\right)$, similar to those of tigecycline (Tables 1 and S2). Considering a similar breakpoint from eravacycline and tigecycline, susceptibility was higher for eravacycline $(65 \%$ vs. $52 \%)$. Nonetheless, the $\mathrm{MIC}_{50 / 90}$ of tigecycline $(0.5 / 1 \mathrm{mg} / \mathrm{L})$ was similar to those of eravacycline, and the difference of percentage of susceptibility was due to a one-MIC dilution difference for the two isolates (Table S2).

\subsubsection{Percentage of Susceptibility According to CLSI Breakpoints}

Different breakpoints to characterise the isolate are observed between the European and US standards. The antibiotics concerned are piperacillin, piperacillin-tazobactam, aztreonam, cefepime, ceftazidime, imipenem, meropenem, ciprofloxacin, levofloxacin, gentamicin, amikacin, tobramycin and SXT (Table S1). For these 13 antibiotics, the MIC value corresponding to the "susceptible" breakpoint is systematically higher in CLSI than in EUCAST. These differences of breakpoints reflect differences concerning percentages of susceptibility. Indeed, the percentage of isolates susceptible to these 13 antibiotics were higher with CLSI than with EUCAST (Table 1). In particular, this concerned levofloxacin (which raised from $4 \%$ to $48 \%$ with CLSI breakpoint), cefepime ( $\leq 10 \%$ from $22 \%$ [Sensititre plate]), ceftazidime (17\% from 30\%), meropenem (48\% from $70 \%$ [Merlin]), amikacin ( $0 \%$ to $22 \%$ [Merlin]) and piperacillin-tazobactam (57\% from $65 \%$ ).

For three antibiotics (meropenem-vaborbactam, tigecycline and eravacycline), no breakpoints were available according to CLSI.

\subsection{Comparison of Different Susceptibility Testing Methods}

Among the 22 antibiotics tested, 11 were evaluated by both Merlin and Sensititre plates. Piperacillin, ceftazidime, ciprofloxacin, levofloxacin, gentamicin and SXT were tested only with the Merlin plate, whereas cefiderocol, imipenem-relebactam, meropenemvaborbactam, tigecycline and eravacycline were evaluated only with the Sensititre plate (Table 1).

We compared the MICs obtained for the 11 antibiotics present in both plates. The agreement between the two microplates varied depending on the antimicrobial agent. An excellent agreement was obtained for piperacillin-tazobactam, aztreonam, ceftolozanetazobactam, fosfomycin, amikacin and tobramycin $(\kappa=1)$ and meropenem $(\kappa=0.91)$, a substantial agreement for ceftazidime-avibactam, cefepime and colistin $(\kappa=0.71,0.65$ and 0.62 , respectively) and a fair agreement for imipenem $(\kappa=0.36)$.

Considering the dilution gap, a perfect match was observed for four of the 11 antibiotics. For six antibiotics, differences in MICs were observed but only with one dilution difference between the two plates, making these discrepancies negligible. In contrast, for three antibiotics (imipenem, meropenem and colistin), these discrepancies involved at least two dilutions (Table 2). The MICs for imipenem were systematically lower in Sensititre plates (five isolates, $2 \mathrm{mg} / \mathrm{L}$ vs. $>8 \mathrm{mg} / \mathrm{L}$ ), while the MICs for meropenem (two isolates) and colistin (one isolate) were lower in Merlin plates (0.5 vs. $2 \mathrm{mg} / \mathrm{L}, 4 \mathrm{vs} .16 \mathrm{mg} / \mathrm{L}$ [meropenem] and $\leq 1 \mathrm{vs.}>16 \mathrm{mg} / \mathrm{L}$ [colistin]) (Table S2). 
Table 2. Concordance between the two microdilution plates (Merlin and Sensititre).

\begin{tabular}{|c|c|c|c|c|c|}
\hline Antimicrobial Agent & $\kappa$ & Perfect Match & 1 Dilution & 2 Dilutions & $>2$ Dilutions \\
\hline Piperacillin-tazobactam & 1 & 23 & & & \\
\hline Aztreonam & 1 & 23 & & & \\
\hline Cefepime & 0.65 & 20 & 3 & & \\
\hline Ceftazidime-avibactam & 0.71 & 20 & 3 & & \\
\hline Ceftolozane-tazobactam & 1 & 21 & 2 & & \\
\hline Imipenem & 0.36 & 19 & & 5 & \\
\hline Meropenem & 0.91 & 20 & 1 & 2 & \\
\hline Colistin & 0.62 & 19 & 3 & & 1 \\
\hline Fosfomycin & 1 & 23 & & & \\
\hline Amikacin & 1 & 22 & 1 & & \\
\hline Tobramycin & 1 & 23 & & & \\
\hline
\end{tabular}

We measured the agreement between the two methods calculating the Cohen's kappa $(\mathrm{k})$, considering susceptibility criteria according to EUCAST breakpoints. We also considered the number of dilution gaps for MIC. For the eight discrepancies ( $\geq 2$ dilutions gap), we performed a third method (E-test ${ }^{\circledR}$ for imipenem and meropenem and UMIC ${ }^{\circledR}$ for colistin). The MICs obtained were between the values of the two plates for imipenem $(6 \mathrm{mg} / \mathrm{L}$ for four isolates and $4 \mathrm{mg} / \mathrm{L}$ for one isolate). For meropenem and colistin, the MICs obtained by the third method were lower than those obtained by Merlin and Sensititre plates ( 0.125 and $2 \mathrm{mg} / \mathrm{L}$ [meropenem], $0.5 \mathrm{mg} / \mathrm{L}$ [colistin]) (Table S2).

The SXT was present exclusively in the Merlin plate but the minimum concentration tested in the plate $(\leq 1 / 19 \mathrm{mg} / \mathrm{L})$ was higher than the EUCAST breakpoint $(\leq 0.125 \mathrm{mg} / \mathrm{L})$. The susceptibility of the SXT was evaluated by disk diffusion as a zone diameter breakpoint is available. A congruence was observed for 21 of the 23 isolates between MIC and zone diameter. In one case, disk diffusion categorised SXT as resistant $(25 \mathrm{~mm}$ [breakpoint $\leq 26 \mathrm{~mm}$ ] whereas MIC was $\leq 1 / 19 \mathrm{mg} / \mathrm{L}$, which did not allow for interpreting the result). In one case, a discrepancy was observed between the two methods $(\mathrm{MIC}=4 \mathrm{mg} / \mathrm{L}$ [resistant], diameter $=28 \mathrm{~mm}$ [susceptible]).

\section{Discussion}

Very few data are currently available about the susceptibility of Achromobacter, particularly with regard to new molecules. We propose here the description of the susceptibility of 23 Achromobacter isolates from PWCF respiratory samples by the determination of MICs of 22 antibiotics active on non-fermenting Gram-negative bacilli. MICs are determined using the broth microdilution method and interpreted according to EUCAST and CLSI.

Among the usual antibiotics, imipenem (70\% susceptibility according to the three methods used), meropenem (43\% [Sensititre], 48\% [Merlin]), piperacillin-tazobactam (57\%), SXT $(65 \%)$ and tigecycline $(52 \%)$ demonstrated the greater activities, according to EUCAST. In contrast, ceftazidime and fluoroquinolones had poor activities $(17 \%$ and $\leq 10 \%$ susceptibility, respectively). Considering CLSI breakpoints, susceptibility raised for levofloxacin ( $4 \%$ to $48 \%$ ), cefepime ( $\leq 10 \%$ to $22 \%$ ), ceftazidime ( $17 \%$ to $30 \%)$, meropenem ( $48 \%$ to $70 \%$ ), amikacin ( $0 \%$ to $22 \%$ ) and piperacillin-tazobactam (57\% to $65 \%)$. Indeed, a large difference was observed according to the reference used, due to differences in breakpoints between the European and US standards. These discrepancies highlight the importance of specific breakpoints to the bacterial genus/species. This lack of specific breakpoints, combined with differences in breakpoints between the European and US standards, makes it difficult to interpret the results. Indeed, although these EUCAST Achromobacter specific breakpoints are currently only covering the top of the iceberg with just three antibiotics, they provide new perspectives essential to guide antimicrobial therapy and improve patient outcomes. Nonetheless, as the publication of specific EUCAST breakpoints is recent, the vast majority of the published data is based on the CLSI breakpoints. Indeed, considering CLSI breakpoints, European studies on the antibiotic susceptibility of Achromobacter isolated from PWCF have shown similar results with a good susceptibility of piperacillin-tazobactam (85 to $89 \%$ ), imipenem (79 to $88 \%$ ) and meropenem (54 to $72 \%$ ) [20-22]. Conversely, US studies showed a lower susceptibility (piperacillin-tazobactam (13 to 55\%), imipenem (59\% and meropenem (28 to $51 \%))[23,24]$. 
We noticed that imipenem was more active than meropenem $\left(\mathrm{MIC}_{50 / 90}=\leq 1 />8\right.$ vs. $2 />16 \mathrm{mg} / \mathrm{L})$ as previously described $[21,23,25]$. These data are in contradiction with the EUCAST, which provides breakpoints for meropenem and proposes that imipenem has intrinsically insufficient activity for the breakpoint to be determined (TECOFF [tentative epidemiological cut-off values] of $8 \mathrm{mg} / \mathrm{L}$ ) [10]. This is also the case for colistin, which shows an interesting activity in our study (39\% susceptibility [Merlin]), and could represent an alternative in the case of pan-drug resistance. This discordance reflects, in our opinion, the lack of data concerning this bacterial genus.

Among the associations of the $\beta$-lactam- $\beta$-lactamase inhibitor, the addition of a $\beta$ lactamase inhibitor does not appear to provide significant improvement. $\mathrm{MIC}_{50}$ and MIC $_{90}$ were observed for $\beta$-lactam alone, as well as the susceptibility percentage were similar to those obtained for $\beta$-lactam- $\beta$-lactamase inhibitor, except for meropenem-vaborbactam and ceftazidime-avibactam. Nonetheless, MIC differences observed between $\beta$-lactam alone and $\beta$-lactam- $\beta$-lactamase inhibitor were slight and concerned generally one MIC dilution. As in our study, Caverly et al. reported no difference between ceftazidime alone and ceftazidime-avibactam and a poor activity of ceftolozane-tazobactam [23]. They showed a better activity of meropenem-vaborbactam than meropenem alone (86\% vs. $72 \%$, breakpoint $\leq 4 \mathrm{mg} / \mathrm{L}$ ) [23]. Given the fact that acquired carbapenem resistance occurs mostly via efflux systems and possibly by metallo- $\beta$-lactamases (MBLs) in Achromobacter species and that vaborbactam has no activity on these two mechanisms, this point needs to be clarified.

Among new therapeutic options, cefiderocol appears to be particularly interesting. Cefiderocol has the advantage of being active on non-fermenting Gram-negative bacilli and of being relatively stable to nearly all $\beta$-lactamase enzymes, including MBLs [26]. In our study, cefiderocol showed activity on all but two isolates ( $91 \%$ susceptibility, $\left.\mathrm{MIC}_{50 / 90}=0.25 / 1 \mathrm{mg} / \mathrm{L}\right)$. In vitro data on cefiderocol activity against Achromobacter are very limited. Rolston et al. defined a $\mathrm{MIC}_{90}=0.125 \mathrm{mg} / \mathrm{L}$ against 15 isolates from cancer patients [27]. This difference in MICs can be explained by at least two factors. The first factor identified is the characteristics of the patients in these studies. Our study concerns PWCF who receive numerous antibiotic therapies, and therefore, Achromobacter isolates are subject to high selective pressure. Secondly, we used a standard cation-adjusted $\mathrm{MH}$ broth, following the manufacturer recommendation for the inoculation of the Sensititre plate and not an iron-depleted MH broth as suggested by EUCAST and CLSI. Cefiderocol relies on active iron transport for entry into the periplasm, which is upregulated under iron-depleted conditions in vivo. Iron-depleted cation-adjusted $\mathrm{MH}$ broth mimics the in vivo condition. Initial studies demonstrated higher MICs when cefiderocol was tested with standard cation-adjusted $\mathrm{MH}$ broth than with iron-depleted broth [28]. Consequently, our MIC values may be overestimated. Concerning in vivo data, the use of cefiderocol in clinical case reports also suggest promising results $[29,30]$.

Concerning eravacycline, a slight difference was noted between eravacycline and tigecycline but concerned only two isolates and one dilution gap. Eravacycline has demonstrated lower MIC than tigecycline for other non-fermenting Gram-negative bacilli as Stenotrophomonas maltophilia, but no Achromobacter isolate was included in the study [31]. Indeed, more studies are required to evaluate eravacycline activity on Achromobacter.

Currently, there are no official recommendations for the treatment of Achromobacter infections. The antibiotic therapy proposed is based on the combination of ciprofloxacin and carbapenem or on the combination of chloramphenicol and minocycline [32]. In our study, although imipenem demonstrated a good activity, all isolates were resistant to ciprofloxacin. Consequently, this association does not seem to be relevant. Both chloramphenicol and minocycline were not evaluated in our study. Due to its toxicity, chloramphenicol is rarely used. We tested tigecycline, a derivative of minocycline, which appears to demonstrate a good activity against Achromobacter $\left(\mathrm{MIC}_{50}=0.5 \mathrm{mg} / \mathrm{L}\right.$ and $1 \mathrm{mg} / \mathrm{L}$ for tigecycline and minocycline, respectively) [33,34]. Overall, clinical studies are needed to make recommendations for the treatment of Achromobacter, and more generally of non-fermenters, 
especially considering the increase in their prevalence and the recent availability of new antimicrobial agents.

As Achromobacter, other non-fermenting Gram-negative bacilli, such as Stenotrophomonas maltophilia, Burkholderia cepacia complex, Pandoraea or Cupriavidus are increasingly isolated from the respiratory tract of PWCF and are characterised by their high resistance to antibiotics. For all these bacteria, SXT and tetracycline (minocycline) are among the most active antimicrobial agents [8,22,34-38]. For most of them, carbapenems represent one of the most active antimicrobial agents except for $S$. maltophilia, which is intrinsically resistant due to a chromosomal carbapenemase. Among newer antimicrobial agents, new $\beta$-lactam$\beta$-lactamase inhibitor associations do not seem to cause any activity for $S$. maltophila or Pandoraea, as we described for Achromobacter spp. [23] (Table 3). However, $\beta$-lactam- $\beta$ lactamase inhibitor seems to be relevant for Burkholderia spp treatment [23]. Cefiderocol exhibits a great activity also against other non-fermenters $[39,40]$. Although clinical studies are needed to confirm the relevance of cefiderocol in the management of non-fermenters infections in CF, this antimicrobial agent seems to represent an interesting alternative to older agents.

Table 3. Activity of newer antimicrobial agents and comparator against non-fermenting Gram-negative bacilli.

\begin{tabular}{|c|c|c|c|c|c|c|c|}
\hline \multirow[b]{2}{*}{ Bacteria } & \multicolumn{7}{|c|}{ Antimicrobial Agent (\% Susceptibility) } \\
\hline & Ceftazidime & $\begin{array}{l}\text { Ceftazidime- } \\
\text { Avibactam }\end{array}$ & $\begin{array}{l}\text { Ceftolozane- } \\
\text { Tazobactam }\end{array}$ & Meropenem & $\begin{array}{c}\text { Meropenem- } \\
\text { Vaborbactam ** }\end{array}$ & Cefiderocol & References \\
\hline Achromobacter spp. & $30-71 \%$ & $26-78 \%$ & $1-10 \%$ & $43-72 \%$ & $65-86 \%$ & $91-97 \%$ & $\begin{array}{l}{[23,36,40,41] ;} \\
\text { Present study }\end{array}$ \\
\hline Burkholderia spp. & $20-91 \%$ * & $24-97 \% *$ & $12-89 \%$ * & $90-100 \%$ & $97-100 \%$ & $75-94 \%$ & {$[23,36,40,41]$} \\
\hline S. malotphilia & $34 \%$ & $27-48 \%$ & $25-27 \%$ & $0-11 \%$ & $0-12 \%$ & $100 \%$ & {$[23,36,40,41]$} \\
\hline Pandorae spp. & $0 \%$ & $0 \%$ & $0 \%$ & $0 \%$ & $0 \%$ & - & {$[23,36,40,41]$} \\
\hline Cupriavidus spp. & $23-27 \%$ & $69-73 \%$ & $73-90 \%$ & $8-18 \%$ & - & - & {$[35,36]$} \\
\hline
\end{tabular}

As great differences exist between the studies (non-species-specific breakpoints, differences between CLSI and EUCAST breakpoints), percentage of susceptibilities are difficult to compare. * Lower susceptibility for B. gladioli, ${ }^{* *}$ breakpoint $\leq 4 \mu \mathrm{g} / \mathrm{mL}$.

In this study, we used two MIC plates in microdilution (Sensititre and Merlin). These two plates were complementary in terms of composition. Some molecules were redundant and allowed us to compare the results of the two plates. In the majority of cases, a perfect match was observed between both plates. Eight mismatches were observed between Merlin and Sensititre plates and concerned carbapenems ( $n=7, k=0.36$ for imipenem) and colistin $(n=1, \mathrm{k}=0.62)$. This can probably be explained, at least in part, by the reading difficulties we encountered. The reading of MICs was sometimes difficult due to the nonhomogeneous appearance of the well, particularly for colistin (Figure S1). In order to limit this bias, the data were systematically read in duplicate. Moreover, colistin has recently been the subject of a warning from EUCAST due to the difficulties of performing MIC determinations. However, the two plates we used were approved by EUCAST and showed good results compared to the reference technique [42]. These reading difficulties could also be responsible for the discrepancies between MIC and the inhibition zone diameters for SXT. Indeed, the fine haze in the inhibition zone made the reading of the diameter around the SXT sometimes difficult.

Several limitations of this study should be acknowledged. First, we tested a limited number of isolates, all from the same CF centre. This study should be confirmed on a larger number of isolates coming from different geographical locations. Second, we did not perform molecular methods to explore the mechanism of resistance or to identify the isolates at the species level. Finally, although we used two MIC plates simultaneously, some molecules were only present in one of the plates, and we did not duplicate the tests.

\section{Conclusions}

The existence of numerous natural and acquired resistances that characterise the Achromobacter genus is associated either with the lack of specific breakpoints or with differences in breakpoints between the European and US standards, making it difficult to interpret the susceptibility results. The recent publication of specific breakpoints by EUCAST provides 
new perspectives essential to guiding antimicrobial therapy and guidelines for the management of Achromobacter infections. Among "older" antimicrobial agents, carbapenems, especially imipenem, piperacillin, SXT and tigecycline are still useful antibiotics for the treatment of Achromobacter infections. Among new therapeutic options, $\beta$-lactams combined with a $\beta$-lactamase inhibitor does not have benefits. On the other hand, cefiderocol appears as a promising therapeutic alternative for managing Achromobacter infections in PWCF. More data, including clinical data, are now required to establish the optimal treatment of Achromobacter infections.

Supplementary Materials: The following are available online at https:/ / www.mdpi.com/article/10 $.3390 /$ microorganisms $9122473 /$ s1, Table S1. The EUCAST and CLSI breakpoints used to interpret Achromobacter MICs in the study; Table S2. MICs values obtained for each antibiotic and for each isolate of this study; Figure S1. Picture of MIC plate result (A) with panel configuration (B) (Merlin) and picture of the inhibition zone diameter for SXT (C).

Author Contributions: Conceptualisation, C.B. and G.H.-A.; methodology, C.B., C.L. and A.M.; formal analysis, C.B., C.L. and A.M.; investigation, S.R., J.L.B., A.B., A.D. and T.R.; resources, C.B., C.L., D.T. and H.L.B.; writing-original draft preparation, C.B. and C.L.; writing-review and editing, C.B., C.L., D.T., H.L.B. and G.H.-A.; supervision, G.H.-A.; project administration, G.H.-A. All authors have read and agreed to the published version of the manuscript.

Funding: This research received no external funding.

Institutional Review Board Statement: Not applicable.

Informed Consent Statement: Not applicable.

Data Availability Statement: Not applicable.

Conflicts of Interest: The authors declare no conflict of interest.

\section{References}

1. Registre Français de la Mucoviscidose Bilan Des Données. 2018. Available online: https://www.vaincrelamuco.org/sites/ default/files/registre_2019_vf.pdf (accessed on 7 September 2021).

2. Marsac, C.; Berdah, L.; Thouvenin, G.; Sermet-Gaudelus, I.; Corvol, H. Achromobacter xylosoxidans airway infection is associated with lung disease severity in children with cystic fibrosis. ERJ Open Res. 2021, 7, 00076-02021. [CrossRef]

3. De Baets, F.; Schelstraete, P.; Van Daele, S.; Haerynck, F.; Vaneechoutte, M. Achromobacter xylosoxidans in Cystic Fibrosis: Prevalence and clinical relevance. J. Cyst. Fibros. 2007, 6, 75-78. [CrossRef] [PubMed]

4. Recio, R.; Brañas, P.; Martínez, M.T.; Chaves, F.; Orellana, M.A. Effect of Respiratory Achromobacter spp. infection on pulmonary function in patients with cystic fibrosis. J. Med. Microbiol. 2018, 67, 952-956. [CrossRef]

5. Tetart, M.; Wallet, F.; Kyheng, M.; Leroy, S.; Perez, T.; Le Rouzic, O.; Wallaert, B.; Prevotat, A. Impact of Achromobacter xylosoxidans isolation on the respiratory function of adult patients with cystic fibrosis. ERJ Open Res. 2019, 5, 00051-02019. [CrossRef] [PubMed]

6. Somayaji, R.; Stanojevic, S.; Tullis, D.E.; Stephenson, A.L.; Ratjen, F.; Waters, V. Clinical outcomes associated with Achromobacter species infection in patients with cystic fibrosis. Ann. Am. Thorac. Soc. 2017, 14, 1412-1418. [CrossRef]

7. Hauser, A.R.; Jain, M.; Bar-Meir, M.; McColley, S.A. Clinical significance of microbial infection and adaptation in cystic fibrosis. Clin. Microbiol. Rev. 2011, 24, 29-70. [CrossRef] [PubMed]

8. Menetrey, Q.; Sorlin, P.; Jumas-Bilak, E.; Chiron, R.; Dupont, C.; Marchandin, H. Achromobacter xylosoxidans and Stenotrophomonas maltophilia: Emerging pathogens well-armed for life in the cystic fibrosis patients' lung. Genes 2021, 12, 610. [CrossRef]

9. Isler, B.; Kidd, T.J.; Stewart, A.G.; Harris, P.; Paterson, D.L. Achromobacter infections and treatment options. Antimicrob. Agents Chemother. 2020, 64, e01025-20. [CrossRef]

10. EUCAST: Consultations. Available online: https://www.eucast.org/publications_and_documents/consultations/ (accessed on 7 September 2021).

11. Pereira, R.H.V.; Leão, R.S.; Carvalho-Assef, A.P.; Albano, R.M.; Rodrigues, E.R.A.; Firmida, M.C.; Folescu, T.W.; Plotkowski, M.C.; Bernardo, V.G.; Marques, E.A. Patterns of virulence factor expression and antimicrobial resistance in Achromobacter xylosoxidans and Achromobacter ruhlandii isolates from patients with cystic fibrosis. Epidemiol. Infect. 2017, 145, 600-606. [CrossRef]

12. Société Française de Microbiologie. Rémic—Référentiel en Microbiologie Médicale, 6th ed.; Société Française de Microbiologie: Paris, France, 2018; pp. 213-220.

13. Spilker, T.; Vandamme, P.; Lipuma, J.J. Identification and distribution of Achromobacter species in cystic fibrosis. J. Cyst. Fibros. 2013, 12, 298-301. [CrossRef]

14. EUCAST: Clinical Breakpoints. Available online: http://www.eucast.org/clinical_breakpoints/ (accessed on 29 October 2018). 
15. EUCAST: Disk Diffusion Methodology. Available online: https://www.eucast.org/ast_of_bacteria/disk_diffusion_methodology / (accessed on 7 September 2021).

16. Schwarz, S.; Silley, P.; Simjee, S.; Woodford, N.; van Duijkeren, E.; Johnson, A.P.; Gaastra, W. Editorial: Assessing the antimicrobial susceptibility of bacteria obtained from animals. J. Antimicrob. Chemother. 2010, 65, 601-604. [CrossRef]

17. CLSI. Performance Standards for Antimicrobial Susceptibility Testing, 31st Edition. Available online: https://clsi.org/standards / products/microbiology/documents/m100/ (accessed on 8 September 2021).

18. Cohen, J. A Coefficient of Agreement for Nominal Scales. Educ. Psychol. Meas. 1960, 20, 37-46. [CrossRef]

19. Landis, J.R.; Koch, G.G. The Measurement of observer agreement for categorical data. Biometrics 1977, 33, 159-174. [CrossRef]

20. Glupczynski, Y.; Hansen, W.; Freney, J.; Yourassowsky, E. In vitro susceptibility of Alcaligenes denitrificans subsp. xylosoxidans to 24 antimicrobial agents. Antimicrob. Agents Chemother. 1988, 32, 276-278. [CrossRef]

21. Amoureux, L.; Sauge, J.; Sarret, B.; Lhoumeau, M.; Bajard, A.; Tetu, J.; Bador, J.; Neuwirth, C.; MucoMicrobes group. Study of 109 Achromobacter spp. isolates from 9 French CF centres reveals the circulation of a multiresistant clone of $A$. xylosoxidans belonging to ST 137. J. Cyst. Fibros. 2019, 18, 804-807. [CrossRef]

22. Coward, A.; Kenna, D.T.D.; Woodford, N.; Turton, J.F.; members of the UK CF Surveillance Working Group. The UK CF Surveillance Working Group comprised. Structured surveillance of Achromobacter, Pandoraea and Ralstonia species from patients in England with cystic fibrosis. J. Cyst. Fibros. 2020, 19, 388-393. [CrossRef]

23. Caverly, L.J.; Spilker, T.; Kalikin, L.M.; Stillwell, T.; Young, C.; Huang, D.B.; LiPuma, J.J. In vitro activities of $\beta$-Lactam- $\beta$ Lactamase inhibitor antimicrobial agents against cystic fibrosis respiratory pathogens. Antimicrob. Agents Chemother. 2019, 64, e01595-19. [CrossRef]

24. Saiman, L.; Chen, Y.; Tabibi, S.; Gabriel, P.S.; Zhou, J.; Liu, Z.; Lai, L.; Whittier, S. Identification and antimicrobial susceptibility of Alcaligenes xylosoxidans isolated from patients with cystic fibrosis. J. Clin. Microbiol. 2001, 39, 3942-3945. [CrossRef]

25. Amoureux, L.; Bador, J.; Siebor, E.; Taillefumier, N.; Fanton, A.; Neuwirth, C. Epidemiology and resistance of Achromobacter xylosoxidans from cystic fibrosis patients in Dijon, Burgundy: First French Data. J. Cyst. Fibros. 2013, 12, 170-176. [CrossRef]

26. Simner, P.J.; Patel, R. Cefiderocol antimicrobial susceptibility testing considerations: The Achilles' heel of the trojan horse? J. Clin. Microbiol. 2020, 59, e00951-20. [CrossRef] [PubMed]

27. Rolston, K.V.I.; Gerges, B.; Shelburne, S.; Aitken, S.L.; Raad, I.; Prince, R.A. Activity of cefiderocol and comparators against isolates from cancer patients. Antimicrob. Agents Chemother. 2020, 64, e01955-19. [CrossRef]

28. Clinical and Laboratory Standards Institute. June, 2017 AST Agenda Summary Minutes; Clinical and Laboratory Standards Institute: Wayne, PA, USA, 2017.

29. Warner, N.C.; Bartelt, L.A.; Lachiewicz, A.M.; Tompkins, K.M.; Miller, M.B.; Alby, K.; Jones, M.B.; Carr, A.L.; Alexander, J.; Gainey, A.B.; et al. Cefiderocol for the treatment of adult and pediatric patients with cystic fibrosis and Achromobacter xylosoxidans infections. Clin. Infect. Dis. 2021, 73, e1754-e1757. [CrossRef]

30. Food and Drug Administration FDA Antimicrobial Drugs Advisory Committee. Cefiderocol Briefing Document; NDA 2094445; Shionogi, Inc.: Florham Park, NJ, USA, 2019. Available online: https:/ /www.fda.gov/media/131705/download (accessed on 29 November 2021).

31. Morrissey, I.; Olesky, M.; Hawser, S.; Lob, S.H.; Karlowsky, J.A.; Corey, G.R.; Bassetti, M.; Fyfe, C. In vitro activity of eravacycline against Gram-negative bacilli isolated in clinical laboratories worldwide from 2013 to 2017. Antimicrob. Agents Chemother. 2020, 64, e01699-19. [CrossRef]

32. Gibson, R.L.; Burns, J.L.; Ramsey, B.W. Pathophysiology and management of pulmonary infections in cystic fibrosis. Am. J. Respir. Crit. Care Med. 2003, 168, 918-951. [CrossRef]

33. Caillon, J.; Lemabecque, V.; Carrere, J.; Pelletier, M.; Batard, E.; Jacqueline, C. In vitro activity of meropenem (MEM), colistin (CS) and tigecycline (TGC) against Achromobacter xylosoxidans (AX) isolated from cystic fibrosis (CF) patients. In Proceedings of the Forty-Ninth Interscience Conference on Antimicrobial Agents and Chemotherapy (ICAAC), San Francisco, CA, USA, 12-15 September 2009.

34. Shortridge, D.; Arends, S.J.R.; Streit, J.M.; Castanheira, M. Minocycline activity against unusual clinically significant Gramnegative pathogens. Antimicrob. Agents Chemother. 2021, 65, e01264-21. [CrossRef] [PubMed]

35. Massip, C.; Coullaud-Gamel, M.; Gaudru, C.; Amoureux, L.; Doleans-Jordheim, A.; Hery-Arnaud, G.; Marchandin, H.; Oswald, E.; Segonds, C.; Guet-Revillet, H. In vitro activity of 20 antibiotics against Cupriavidus clinical strains. J. Antimicrob. Chemother. 2020, 75, 1654-1658. [CrossRef]

36. Massip, C.; Mathieu, C.; Gaudru, C.; Miaut, V.; Floch, P.; Oswald, E.; Segonds, C.; Guet-Revillet, H. In vitro activity of seven $\beta$-lactams including ceftolozane/tazobactam and ceftazidime/avibactam against Burkholderia cepacia complex, Burkholderia gladioli and other non-fermentative Gram-negative bacilli isolated from cystic fibrosis patients. J. Antimicrob. Chemother. 2019, 74, 525-528. [CrossRef] [PubMed]

37. Capaldo, C.; Beauruelle, C.; Saliou, P.; Rault, G.; Ramel, S.; Héry-Arnaud, G. Investigation of Stenotrophomonas Maltophilia Epidemiology in a French Cystic Fibrosis Center. Respir. Med. Res. 2020, 78, 100757. [CrossRef]

38. Gajdács, M.; Urbán, E. Prevalence and antibiotic resistance of Stenotrophomonas maltophilia in respiratory tract samples: A 10-year epidemiological snapshot. Health Serv. Res. Manag. Epidemiol. 2019, 6, 1-9. [CrossRef] 
39. Naas, T.; Lina, G.; Santerre Henriksen, A.; Longshaw, C.; Jehl, F. In vitro activity of cefiderocol and comparators against isolates of Gram-negative pathogens from a range of infection sources: SIDERO-WT-2014-2018 Studies in France. JAC Antimicrob. Resist. 2021, 3, dlab081. [CrossRef]

40. Gavioli, E.M.; Guardado, N.; Haniff, F.; Deiab, N.; Vider, E. Does cefiderocol have a potential role in cystic fibrosis pulmonary exacerbation management? Microb. Drug Resist. 2021. Online ahead of print. [CrossRef] [PubMed]

41. Gant, V.; Hussain, A.; Bain, M.; Longshaw, C.; Henriksen, A.S. In vitro activity of cefiderocol and comparators against gramnegative bacterial isolates from a series of surveillance studies in England: 2014-2018. J. Glob. Antimicrob. Resist. 2021, 27, 1-11. [CrossRef] [PubMed]

42. EUCAST: Warnings! Available online: https://www.eucast.org/ast_of_bacteria/warnings/ (accessed on 10 September 2021). 